\title{
Ultrasound Feature Evaluation for Robustness to Sensor Shift in Ultrasound Sensor Based Hand Motion Recognition.
}

\author{
Peter Boyd, Yinfeng Fang, and Honghai Liu \\ School of Computing, \\ University of Portsmouth, \\ Portsmouth, United Kingdom \\ peter.boyd@port.ac.uk \\ yinfeng.fang@port.ac.uk \\ honghai.liu@port.ac.uk
}

\begin{abstract}
Pattern Recognition based approaches have offered great promise in the field of bio-signal controlled prosthesis. Traditionally Surface Electromyography based Approaches (SEMG) have been used to satisfy the purpose of providing Bio-Signal control in upper extremity Prosthesis. Although these methods have been shown to be robust, there still exists issues in performance within clinical environments. In recent years, Ultrasound signal based methods have seen growing interest within the field of motion Recognition, largely due to the increased resolution, deeper muscle observation, and reduced cross-talk that can be achieved in comparison to SEMG methods. However, the methods to be applied for hand Motion recognition are still only just beginning to be explored. In this paper, we shall investigate the applicability of SEMG feature extraction techniques to Ultrasound based hand motion recognition and the subsequent impact of Sensor shift on these features. The results of this study indicate that SEMG feature extraction techniques have excellent single location accuracy in Ultrasound based Hand motion recognition. However this paper more visibly presents the strong impact of Sensor Shift on A-Mode ultrasound based hand motion Recognition, and finally presents which feature extraction methods are most robust to this shift.
\end{abstract}

Keywords: Ultrasound · Hand Gesture Recognition · Feature Extraction · Sensor Shift · Prosthesis · Pattern Recognition.

\section{Introduction}

Bio-Signal controlled prosthesis are highly important to enabling amputees to be capable of mitigating the impact on their quality of life that comes with a lost limb [3]. Whereas early prosthesis had either limited or just no functionality, modern prosthesis have seen promising growth in providing a more intuitive control system for amputees. Generally speaking, a bio-signal controlled prosthesis attempts to relate a given set of bio-input to a set of anticipated motions. The 
exact forms of bio-signal controlled upper limb prosthesis can be divided into two categories, conventional bio-signal prosthesis or pattern recognition based bio-Signal control strategies [17]. The most common form of these bio-signal controlled devices comes in the form of Electromyography (EMG) based bio-signal control devices. Traditionally conventional EMG controlled prosthesis followed an "on-off" switched control system, however newer conventional devices provide a more diverse series of control routines and inputs [16]. The benefit of these conventional approaches are that they are simple to implement, provide a desirable degree of robustness to transient changes in the bio-signal, and can satisfy the basic needs of an amputee for daily use. Conversely, conventional bio-signal controlled prosthesis hold limitations in several areas, such as having limited degrees of functionality and most importantly that their control scheme is unnatural in comparison to how a person would naturally move their original limb prior to amputation. Pattern Recognition based approaches, however, follow in the concept that an amputee may be able to voluntarily produce repeatable bio-signals that can be directly mapped to gestures that are best fitting to that bio-signal. Therefore, pattern recognition based approaches have seen considerable growth of interest in academia in recent years as they promise control schemes which are seemingly more natural to an amputee, whilst also providing a potentially larger pool of gestures that an amputee can perform therefore allowing a larger increase in quality of life, and finally this natural control scheme may aid in reducing the cognitive burden involved in the rehabilitative procedure of training the amputee with their new prosthesis.

\subsection{Transient Changes}

As with Conventional approaches, Pattern Recognition devices that utilize EMG have seen much popularity within academia, presently demonstrating highly promising results within laboratory environments. Unfortunately, EMG based pattern recognition approaches experience varying issues in their viability once applied to a clinical scenario. As for how such a dichotomy may occur a specific set of transient changes within Pattern Recognition based control systems have been cited in literature, these issues being electrode shift [20], crosstalk, fatigue [12], changes to skin conductivity, time[2,10,4], and concept drift.

\subsection{Ultrasound}

In an attempt to explore robust alternatives to SEMG motion recognition, researchers have in recent years began investigating the applicability of ultrasound sensors for the purpose of motion recognition [1] [19]. With good promise being displayed in the topics of rehabilitative Human Machine Interaction [7]. Researchers have noted that the higher resolution of Ultrasound Signal and ability to observe deeper tissue than SEMG sensors as factors that could provide more robust control schemes [18]. The usage Ultrasound (US) imaging has long been used as a non invasive method for visualizing the inside of a body. The method of action for US Imaging, in its most simple form, is by projecting a beam of high 
frequency sound waves from a piezoelectric transducer, where the subsequent echoes of this sound wave can then be monitored for intensity and amount of time it took for the echo to return. This common usage of ultrasound for imaging in the medical field can be tied to the capability of the ultrasonic sound waves to penetrate soft tissues without harming them, a trait long previously recognised by [6]. Typically, Ultrasound methods used for hand motion recognition will either be the more traditional B-Mode Ultrasound, or the somewhat more compact but low resolution A-Mode Ultrasound.

\subsection{Area of focus - Electrode / Diode shift}

As with sEMG based hand motion recognition, ultrasound diode shift may drastically impact the quality of long term hand motion recognition. Frequently with sEMG devices, a small degree of electrode shift may have an insignificant impact on the classifiers performance. The likely reasoning for this being due to area of detection of sEMG electrodes to be shallow but across a larger area, frequently meaning that the main impact of electrode shift comes in the form of reduced amplitude of the targeted muscle and increased crosstalk form neighbouring muscles. It had also been observed that shift may impact SEMG signals dependent upon if the shift is perpendicular or parallel to the original location [9].

With a-mode ultrasound, the issue of shift manifests itself in a much more noticeable fashion. It could best be considered to be from the area targeted by a-mode ultrasound diodes to be deeper than sEMG electrodes but also much more concentrated, effectively removing much of the crosstalk but providing very different signals dependant upon the sensors location.

Although it is an easy argument to make that the quality of a-mode ultrasound will be affected heavily by ultrasound shift, it is important that we quantify just what degree of performance impact can be expected from such shift such that we may make progress towards counteracting shift.

In this paper, we will firstly define the problem of a-mode ultrasound diode shift and how this shift may manifest itself in the visible signal, secondly we will quantify the relationship between degrees of ultrasound diode shift across a targeted area of the forearm and degradation of classifier performance that comes with shift, we shall explore available feature extraction methods and feature sets.

Thirdly, the relative performance of these Feature sets shall be compared for their classification accuracy and rate of degradation across increasing shift.

\section{Materials and methods}

\subsection{Ultrasound Data Collection Device}

The ultrasound data was collected through a 2 channel A-Mode Ultrasound device that collected 100 data points (or time dots) at a rate of 10MHZ. The device was placed on the muscle grouping above and below the wrist of the candidate. Transmission of Ultrasound data was performed via Ethernet to a windows based PC. 


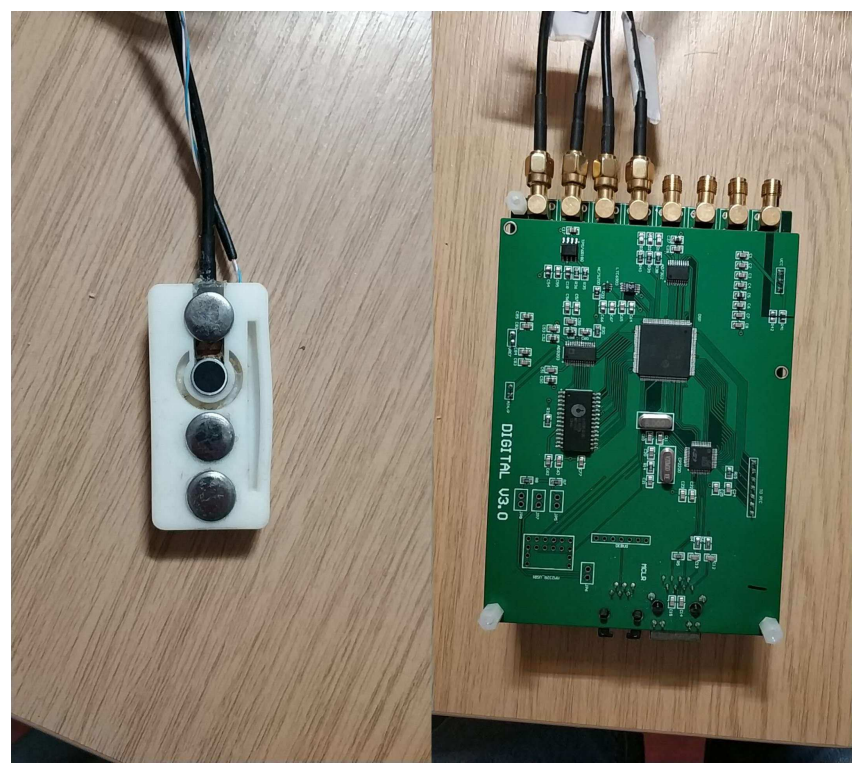

Fig. 1. Ultrasound collection hardware

\section{$2.2 \quad$ Representing Shift}

In order to correctly model the degree of shift across the targeted muscle area, a 7 by 7 grid of 49 individual locations was marked on the top and bottom of the subjects forearm. This set location of space was chosen to simulate both the potential shift from extended daily use, and the expected shift from donning and doffing the ultrasound device.

\subsection{Experimental protocol}

For this preliminary study, a single able bodied participant was used. The participant had prior experience with SEMG motion recognition. for each set of data collected, the participant would be advised to follow a set of hand motion gestures in sequence through on-screen guidance. After every set of motions was completed, both the upper and lower Ultrasound diode would be moved to a new location with ultrasound gel reapplied as necessary.

\subsection{Motions}

The motion collection scheme consisted of 6 gestures that involved either movement of the wrist or hand. The selected gestures were hand at rest, hand open, hand closed, wrist flexion, wrist extension, fine pinch. Each gesture was performed sequentially, for a period of 10 seconds per gesture before shifting to the 
next gesture. A period of 5 minutes was provided between locational shifts as to prevent fatigue.

The motion collection scheme consisted of several minor and major movements involving the hand or entire arm referred to as a primitive. For every collected dataset, a particular motion and its opposite would be performed sequentially, before a period of resting time. The allocated time per gesture was 5 seconds each, with a 10 second resting period. As each set of primitives were repeated 5 times, the resulting dataset would feature 110 seconds of data with 50 seconds of motion activity.

\subsection{Data Pre-Processing}

All data processing was completed using Matlab r2017b. The pre processing for the ultrasound data firstly saw 6 seconds of stable motion data from each 10 second gesture performed, by removing 2 seconds from the beginning and end of each gesture. The intention of trimming using only the stable motion data is to the starting and ending 20 time points of each frame of data as the information carried here was not considered meaningful. A hilbert transform was subsequently applied onto the trimmed data and the envelope was extracted when viable for the chosen feature.

As the A-mode ultrasound data consists of a single frame every $100 \mathrm{~ms}$ containing 960 time points, these time points indicating the muscle activity at a given depth from the US Diode. Presently, as there exists little comparative US feature selection strategies or comparisons. Therefore, traditional feature extraction methods for EMG data were to be modified to better exploit the generalizable traits of the data. The approach to feature extraction was to operate directly on the time points within each frame, as opposed to across multiple frames, using a $120 \mathrm{~ms}$ window and a $30 \mathrm{~ms}$ sliding window.

\subsection{Data Processing}

As mentioned in the pre-processing stage, few feature selection methods for AMode Ultrasound have been evaluated in literature. Therefore this paper shall explore the applicability of of several TD-AR methods in both the no shift and the shift conditions.

To evaluate the quality of the feature sets, several simple features shall be selected, these being Root Mean Square (RMS), Auto Regressive Coefficients (AR), Waveform Transform (WL), Slope Sign Change(SSC), Mean + Standard Deviation (MSD), Zero Crossing (ZC), and Mean Absolute Value (MAV), all of these being traditional EMG feature extraction methods that had been implemented frequently in literature.

for classification, LDA, was used, due to this being suggested as a method that is robust to changes in input signal [11], alongside performing well during Ultrasound Hand Motion Recognition compared to methods such as Decision trees [15]. 


\section{Methodology}

During normal data collection from any bio-signal based system, the sensor is placed on the optimal location for any data to be collected. While this may provide the best exemplar of the expected bio-signal, it can be expected that any external sensor will shift from this location during daily use. Therefore, it was chosen to exploit the unforeseeable directionality of this diode shift by comparing the decreased performance of a trained classifier from the nominal training location in any given direction for every feature set. The working theory behind this selection is to provide the best characteristic of minor or major shift within a 360 degree field around the nominal location. For each location, a dataset was trained, and then tested against all neighbouring nodes. A benefit of this approach is that while there may be an individual subject the quantity of data and classifications for each individual location will bolster the potential for variability within the datasets.

\section{Results}

In fig.4, the outputs of a 10kfold loss method are demonstrated for each set of single Feature case, as an average of all 49 locational datasets. Generally, the performance of each method provides very good responses for when there is no shift in the Ultrasound sensor. However, once shift is applied, then the rate of accuracy for all cases begin to drop at a considerable rate, as shown in fig2.

One noticeable change between the two charts is where Zero Crossing and Slope Sign change perform the worst under no shift, however, once shift is applied then both methods achieve not only a higher base accuracy, but also degrade at a similar but reduced rate in comparison to other methods.

In Table 1, the spatial performance of all single features are displayed, alongside the top five of each increasingly large feature set upto size for features $=5$, then the top two sets of six feature featuresets, and finally the featureset of all features together. The most visible trait of this table is that each subsequent set of combined features perform worse than the prior best performing features, such as SSC-ZC out performing the WL-SSC-ZC combination.

\section{Discussion}

In this study, several feature extraction methods were analysed to observe their robustness to Ultrasound Sensor Shift. The results of this study demonstrated that, while ultrasound based methods achieve good accuracy when kept on the nominal location, there is a significant impact on performance from shift, especially when larger than $4 \mathrm{~mm}$ shift. this would appear to be very much in-line with the findings of other researchers when experiencing transducer shift [5, 19], alongside similar reports being found in SEMG signal pattern recognition[21]. One likely factor into the heavier degrade in accuracy seen in this study is due to the nature of A-Mode Ultrasound having a deeper but much more concentrated 


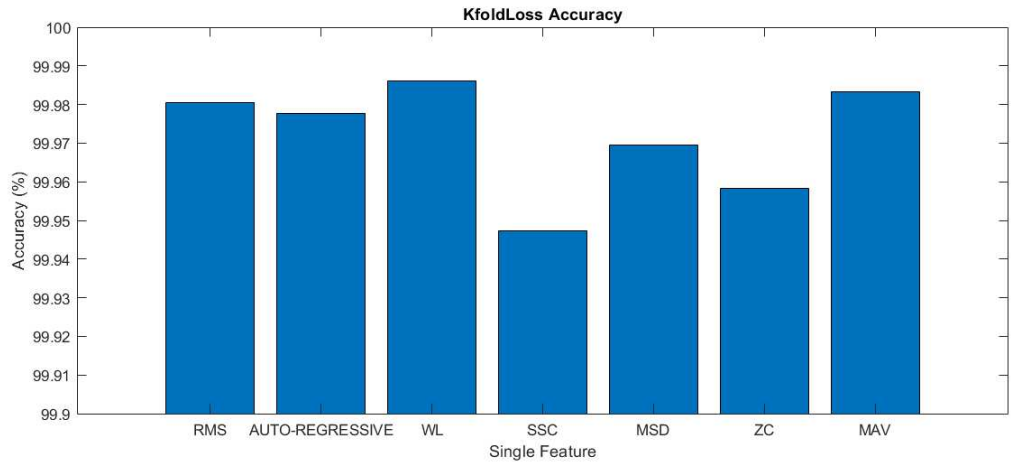

Fig. 2. Relative KfoldLoss Accuracy from each single Feature method

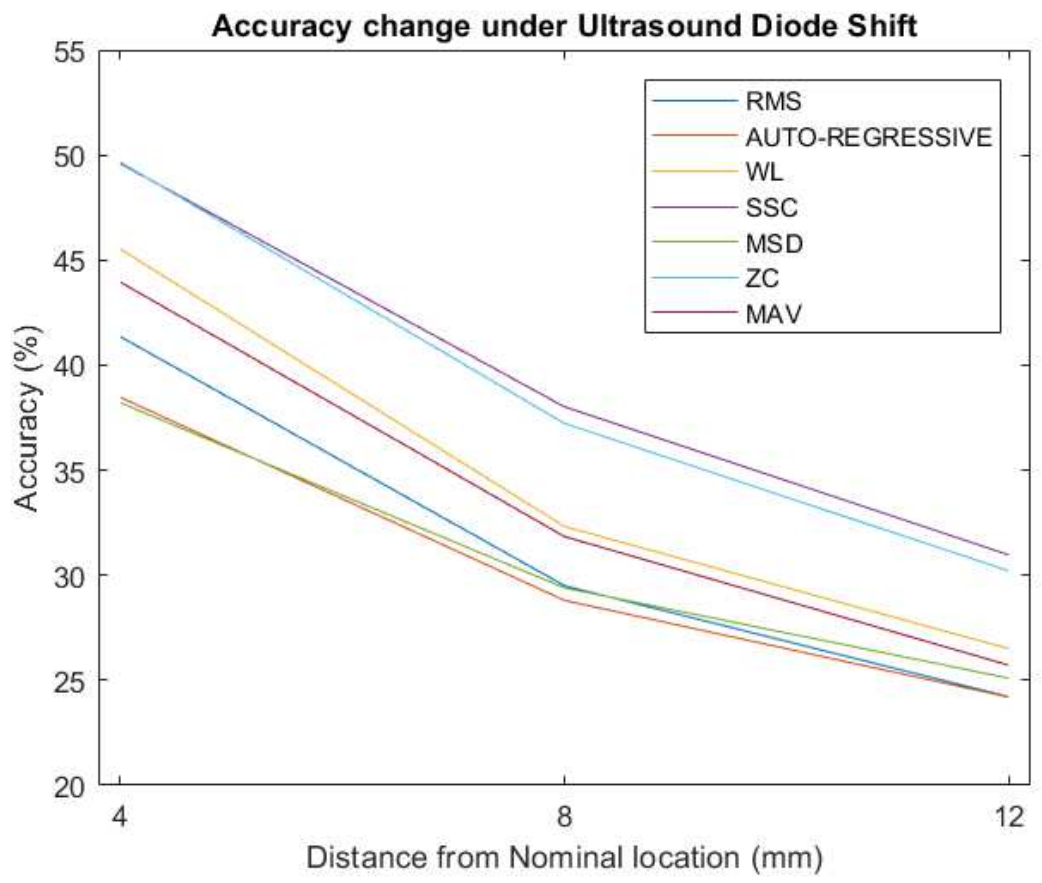

Fig. 3. Change in Accuracy under Ultrasound Sensor Shift conditions 
Table 1. Comparison of Feature Extraction techniques and Feature sets under sensor Shift

\begin{tabular}{|c|c|c|c|}
\hline \multirow{2}{*}{ Feature } & \multicolumn{3}{|c|}{ Accuracy } \\
\hline & $4 \mathrm{~mm}$ & $8 \mathrm{~mm}$ & $12 \mathrm{~mm}$ \\
\hline Root Mean Square (RMS) & 41.3502 & 29.4994 & 24.2456 \\
\hline AutoRegressive (AR) & 38.4634 & 28.8036 & 24.2031 \\
\hline Waveform Length (WL) & 45.5218 & 32.3189 & 26.5053 \\
\hline Slopesign Change (SSC) & 49.5948 & 38.0151 & 30.9701 \\
\hline Mean + Standard Deviation (MSD) & 38.2035 & 29.3982 & 25.1040 \\
\hline Zero Crossing (ZC) & 49.6582 & 37.2260 & 30.2115 \\
\hline Mean Absolute Value (MAV) & 43.9495 & 31.8411 & 27.7215 \\
\hline $\mathrm{SSC}-\mathrm{ZC}$ & 49.2386 & 37.2020 & 30.6760 \\
\hline WL-ZC & 45.7314 & 32.4774 & 26.5321 \\
\hline WL-SSC & 44.8713 & 32.3658 & 26.2964 \\
\hline ZC-MAV & 44.1881 & 32.0853 & 26.0027 \\
\hline WL-MAV & 43.9709 & 31.5118 & 28.9517 \\
\hline WL-SSC-ZC & 44.8788 & 31.6372 & 25.9495 \\
\hline SSC-ZC-MAV & 43.7783 & 31.6879 & 25.7942 \\
\hline WL-ZC-MAV & 43.5127 & 31.1777 & 25.6881 \\
\hline WL-SSC-MAV & 43.1884 & 31.3576 & 26.1230 \\
\hline WL-MSD-ZC & 43.1755 & 31.5351 & 25.9677 \\
\hline SSC-MSD-ZC-MAV & 42.2245 & 30.0888 & 25.3892 \\
\hline WL-MSD-ZC-MAV & 42.0067 & 30.1947 & 24.7848 \\
\hline WL-SSC-MSD-ZC & 41.9771 & 30.5604 & 25.4144 \\
\hline WL-SSC-MSD-MAV & 41.9579 & 30.5913 & 25.0622 \\
\hline WL-SSC-ZC-MAV & 41.4526 & 30.4854 & 25.6745 \\
\hline AUTO-REGRESSIVE-WL-SSC-ZC-MAV & 42.6839 & 30.8301 & 25.3570 \\
\hline AUTO-REGRESSIVE-WL-SSC-MSD-ZC & 42.3035 & 31.3956 & 25.6678 \\
\hline RMS-AUTO-REGRESSIVE-SSC-ZC-MAV & 41.9753 & 30.3633 & 25.1596 \\
\hline AUTO-REGRESSIVE-SSC-MSD-ZC-MAV & 41.9150 & 30.0390 & 24.9601 \\
\hline RMS-AUTO-REGRESSIVE-WL-SSC-ZC & 41.8352 & 31.0312 & 25.4697 \\
\hline AUTO-REGRESSIVE-WL-SSC-MSD-ZC-MAV & 43.7954 & 32.0128 & 26.3671 \\
\hline RMS-AUTO-REGRESSIVE-WL-SSC-MSD-ZC-MAV & 43.7206 & 32.5627 & 26.7492 \\
\hline
\end{tabular}


section of the arm to observe, in a sense it could be considered that the resilience to crosstalk also makes Ultrasound methods less resilient to shift. Other factors that could potentially negatively impact the performance of a Ultrasound based method is that of the subject unconsciously moving their wrist, or fingers wither when performing a movement or by not fully returning to a rest state, as noted by Li [13]. Further to this, the grip strength used in a grasp may also deteriorate a classifier, as noted by Ortenzi et al [15]. However, the most likely major factor here is directly due to the manifestation of shift itself and therefore the focus is on what feature sets may be robust to this issue.

One notable trait of the results displayed here is that the multiple feature datasets all gradually performed worse as more features were added. This could imply a degree of over training is occurring from the single nominal location datasets. In all cases, Zero Crossing, Slope sign change, and Waveform Length, constituted the three main features in any strong dataset. Therefore, it could be described that these 3 features are far more robust to the occurrence of Sensor shift in Ultrasound hand, otion recognition. This could suggest that these methods are stronger at defining the spikes within the ultrasound signal, while other approaches are likely to smooth the signal and bring forward minor changes in signal that don't relate directly to expected signal. Moving forward, there could be great promise in combining these existing features with methods that promote retraining, or the construction of robust datasets that anticipate the impact of shift or other transient changes in signal $[14,8]$.

A further consideration is that the results displayed here are on a two channel system, whilst prior studies used a 4 channel device. It is likely that a 4 channel device could only serve to further improve the accuracy demonstrated in this study.

\section{Conclusion}

In this paper, the robustness of traditional EMG feature extraction techniques were explored when applied to Ultrasound Sensor based Hand motion recognition.From the results of this study, there are two major conclusions. Firstly: the main feature Extraction methods demonstrated that the quality of Ultrasound based hand motion recognition is extremely high under no shift conditions. However, it can be recognised that Ultrasound sensor shift can significantly impact the quality of the classification result, especially at larger ranges.

Secondly, the results that are demonstrated in this paper indicate that combined feature sets seemingly do not perform as well as individual features. This could suggest that the matter comes to being related specifically to the representation of the ultrasound data, or potentially as a consequence of over training in the cases of combined feature sets.

The intent of this research was to explore the relative robustness of different Ultrasound feature extractions methods under the situation of sensor shift and to find a feature or set of features that can provide a meaningful representation of the Ultrasound Signal for hand motion recognition. 
It is suggested that future directions in Ultrasound hand motion recognition is to investigate whether the inclusion of more channels when considering sensor shift may further improve the classification accuracy alongside the impact of larger arm motions. To expand the features tested here on other traditional feature selection methods. Further to this, to investigate training strategies that may improve the robustness to sensor shift in Ultrasound. Finally, it is suggested to further investigate the relative comparison of Ultrasound based hand Motion Recognition, in comparison to EMG when considering Long Term Use.

\section{References}

1. Akhlaghi, N., Baker, C.A., Lahlou, M., Zafar, H., Murthy, K.G., Rangwala, H.S., Kosecka, J., Joiner, W.M., Pancrazio, J.J., Sikdar, S.: Real-time classification of hand motions using ultrasound imaging of forearm muscles. IEEE Transactions on Biomedical Engineering (2016). https://doi.org/10.1109/TBME.2015.2498124

2. Amsuss, S., Paredes, L.P., Rudigkeit, N., Graimann, B., Herrmann, M.J., Farina, D.: Long term stability of surface EMG pattern classification for prosthetic control. In: Proceedings of the Annual International Conference of the IEEE Engineering in Medicine and Biology Society, EMBS. pp. 3622-3625 (2013). https://doi.org/10.1109/EMBC.2013.6610327

3. Biddiss, E.a., Chau, T.T.: Upper limb prosthesis use and abandonment: a survey of the last 25 years. Prosthetics and orthotics international 31(3), 236-257 (2007). https://doi.org/10.1080/03093640600994581

4. Boyd, P., Fang, Y., Liu, H.: Preliminary results of emg-based hand gestures for long term use. In: Huang, Y., Wu, H., Liu, H., Yin, Z. (eds.) Intelligent Robotics and Applications. pp. 98-108. Springer International Publishing, Cham (2017)

5. Castellini, C., Passig, G., Zarka, E.: Using ultrasound images of the forearm to predict finger positions. IEEE Transactions on Neural Systems and Rehabilitation Engineering 20(6), 788-797 (2012). https://doi.org/10.1109/TNSRE.2012.2207916

6. Donald, I., Macvicar, J., Brown, T.G.: INVESTIGATION OF ABDOMINAL MASSES BY PULSED ULTRASOUND. The Lancet (1958). https://doi.org/10.1016/S0140-6736(58)91905-6

7. González, D.S., Castellini, C.: A realistic implementation of ultrasound imaging as a human-machine interface for upper-limb amputees. Frontiers in Neurorobotics (2013). https://doi.org/10.3389/fnbot.2013.00017

8. Hargrove, L., Englehart, K., Hudgins, B.: A training strategy to reduce classification degradation due to electrode displacements in pattern recognition based myoelectric control. Biomedical Signal Processing and Control (2008). https://doi.org/10.1016/j.bspc.2007.11.005

9. He, J., Sheng, X., Zhu, X., Jiang, N.: Electrode density affects the robustness of myoelectric pattern recognition system with and without electrode shift. IEEE Journal of Biomedical and Health Informatics (2019). https://doi.org/10.1109/JBHI.2018.2805760

10. Kaufmann, P., Englehart, K., Platzner, M.: Fluctuating EMG signals: Investigating long-term effects of pattern matching algorithms. In: 2010 Annual International Conference of the IEEE Engineering in Medicine and Biology Society, EMBC'10 (2010). https://doi.org/10.1109/IEMBS.2010.5627288 
11. Kaufmann, P., Englehart, K., Platzner, M.: Fluctuating EMG signals: Investigating long-term effects of pattern matching algorithms. In: 2010 Annual International Conference of the IEEE Engineering in Medicine and Biology Society, EMBC'10 (2010). https://doi.org/10.1109/IEMBS.2010.5627288

12. Lalitharatne, T.D., Hayashi, Y., Teramoto, K., Kiguchi, K.: Compensation of the effects of muscle fatigue on EMG-based control using fuzzy rules based scheme. In: Proceedings of the Annual International Conference of the IEEE Engineering in Medicine and Biology Society, EMBS. pp. 6949-6952 (2013). https://doi.org/10.1109/EMBC.2013.6611156

13. Li, Y., He, K., Sun, X., Liu, H.: Human-machine interface based on multi-channel single-element ultrasound transducers: A preliminary study. In: 2016 IEEE 18th International Conference on e-Health Networking, Applications and Services, Healthcom 2016 (2016). https://doi.org/10.1109/HealthCom.2016.7749483

14. Liu, J., Sheng, X., Zhang, D., Jiang, N., Zhu, X.: Towards Zero Retraining for Myoelectric Control Based on Common Model Component Analysis. IEEE Transactions on Neural Systems and Rehabilitation Engineering (2016). https://doi.org/10.1109/TNSRE.2015.2420654

15. Ortenzi, V., Tarantino, S., Castellini, C., Cipriani, C.: Ultrasound imaging for hand prosthesis control: A comparative study of features and classification methods. In: IEEE International Conference on Rehabilitation Robotics (2015). https://doi.org/10.1109/ICORR.2015.7281166

16. Scheme, E., Englehart, K.: Electromyogram pattern recognition for control of powered upper-limb prostheses: State of the art and challenges for clinical use. Journal of Rehabilitation Research and Development 48(6), 643-660 (2011). https://doi.org/10.1682/JRRD.2010.09.0177, http://www.rehab.research.va.gov/jour/11/486/pdf/scheme486.pdf

17. Toledo, C., Simon, A., Muñoz, R., Vera, A., Leija, L., Hargrove, L.: A comparison of direct and pattern recognition control for a two degree-of-freedom above elbow virtual prosthesis. In: Proceedings of the Annual International Conference of the IEEE Engineering in Medicine and Biology Society, EMBS (2012). https://doi.org/10.1109/EMBC.2012.6346925

18. Yang, X., Li, Y., Fang, Y., Liu, H.: A preliminary study on the relationship between grip force and muscle thickness. In: International IEEE/EMBS Conference on Neural Engineering, NER. pp. 118-121 (2017). https://doi.org/10.1109/NER.2017.8008306

19. Yang, X., Sun, X., Zhou, D., Li, Y., Liu, H.: Towards wearable amode ultrasound sensing for real-time finger motion recognition. IEEE Transactions on Neural Systems and Rehabilitation Engineering (2018). https://doi.org/10.1109/TNSRE.2018.2829913

20. Young, A.J., Hargrove, L.J., Kuiken, T.a.: The effects of electrode size and orientation on the sensitivity of myoelectric pattern recognition systems to electrode shift. IEEE Transactions on Biomedical Engineering 58(9), 2537-2544 (2011). https://doi.org/10.1109/TBME.2011.2159216

21. Young, A.J., Hargrove, L.J., Kuiken, T.A.: Improving myoelectric pattern recognition robustness to electrode shift by changing interelectrode distance and electrode configuration. IEEE Transactions on Biomedical Engineering (2012). https://doi.org/10.1109/TBME.2011.2177662 